\title{
Degludec - First of a New Generation of Insulins
}

\author{
Anja De Rycke ${ }^{1}$ and Chantal Mathieu ${ }^{2}$
}

1. Research Fellow; 2. Professor of Medicine, Department of Endocrinology, University Hospitals Leuven

\begin{abstract}
Diabetes is associated with several serious chronic complications, impacting quality of life and health costs and decreasing survival. These complications can be reduced by obtaining an adequate glycaemic control. The use of current insulin preparations, however, is associated with many disadvantages, leading to suboptimal use and hence decreased efficacy. Therefore, there is an urgent need for new types of basal insulin analogues, providing a very stable and long basal insulin supply. Degludec and Degludecplus, a combination of degludec and a short-acting insulin analogue, are the first developed new insulin formulations fulfilling these criteria. Promising Phase II and preliminary Phase III data have shown that this new therapy is both efficacious and safe. In combination with a healthy lifestyle, this new generation of insulin might become the new standard of care in type 1 and 2 diabetes, reducing both fatal and non-fatal complications and improving quality of life.
\end{abstract}

\section{Keywords}

Compliance, degludec, degludecplus, diabetes, hypoglycaemia, insulin, nocturnal hypoglycaemia, peakless, quality of life, subcutaneous injection

Disclosure: Anja De Rycke has no conflicts of interest to declare. Chantal Mathieu is an adviser for Novo Nordisk and sanofi-aventis. Received: 14 July 2011 Accepted: 5 September 2011 Citation: European Endocrinology, 2011;7(2):84-7 DOI:10.17925/EE.2011.07.02.84 Correspondence: Chantal Mathieu, Department of Endocrinology, University Hospitals Leuven, Herestraat 49, 3000 Leuven, Belgium. E: Chantal.mathieu@uzleuven.be

\section{The Need for New Insulin Analogues}

Diabetes is associated with several serious chronic complications, including nephropathy, retinopathy, neuropathy and most importantly cardiovascular disease, impacting quality of life and health costs and decreasing survival. These complications can be reduced both in type 1 and type 2 diabetes patients, by obtaining an adequate glycaemic control. However, this main goal of diabetes management is only reached in a minority of patients because of several patient-, physician- and treatment-related factors. Insulin is the cornerstone of treatment in type 1 diabetes, but due to the progressive nature of the disease, also the majority of type 2 diabetic patients will eventually need insulin in their treatment. However, delay in insulin initiation is still a major issue in type 2 diabetes and in both type 1 and type 2 insulin-treated patients, adherence to strict insulin regimens and insulin intensification remain a major problem for several reasons. These include injection related issues, systemic side effects (hypoglycaemia, weight gain), psychological reasons, costs and fear for deterioration of quality of life because of the strict and complex schedules of the current insulin regimens.

Besides global glycaemic control, the occurence of severe hypoglycaemic episodes as a cause of morbidity and mortality recently has emerged as a controversial issue in management of type 2 diabetes patients. This controversy was illustrated by the disturbing results of three recent large prospective trials in type 2 diabetes patients. In these trials patients were randomised to receive standard therapy or intensive therapy and hypoglycaemic events were related to adverse outcomes. In the Action to control cardiovascular risk in diabetes (ACCORD) trial the intensive therapy arm was discontinued after a mean of 3.5 years of follow up due to the finding of a higher mortality rate compared to the standard arm. Although explanatory analyses did not reveal an explanation for this excess mortality, the higher rate of severe hypoglycaemic episodes (16.2\% versus $5.1 \%$ ) in the intensive treatment group remained a possible culprit. However, in the other trials, no difference in mortality was seen between standard and intensive treatment, although the incidence of severe hypoglycaemic episodes (21.2\%) was even higher in one study (the Veterans Affairs diabetes trial, [VADT]). Even if hypoglycaemia does not impact different vascular and non-vascular endpoints, reducing its rate remains very important from a patient's perspective as repetitive hypoglycaemic episodes are adversely associated with psychosocial integration and quality of life..$^{1,2,3}$

Here we discuss a new basal insulin, with a novel mechanism of protraction, suggesting a very stable, very long basal insulin supply. Access to a good basal insulin is essential for type 1 diabetic patients using multiple daily injections (MDI), as 24-hour coverage is needed. Indeed, just like the native beta-cells provide continuous small basal levels of insulin to prevent reverting from anabolism to catabolism, also type 1 diabetic patients need perfect coverage to prevent 'escape' phenomena by the next basal injection. However, also in type 2 diabetes, access to a better basal insulin is needed. Basal insulin supplementation is the cornerstone of adequate glycaemic control in type 2 diabetic patients experiencing suboptimal oral therapy. Besides classical Neutral Protamine Hagedorn (NPH) insulin, two long-acting basal insulin analogues, detemir and glargine, are available. In contrast to NPH insulin, which shows a pronounced insulin peak six to eight hours following administration, long-acting analogues are characterised by a longer duration of action, limiting the peak effect and hence reducing the risk for hypoglycaemia 
without compromising glycaemic control. In addition, within-subject variability is less pronounced compared to classical NPH insulin. However, these new types of insulin analogues still have major limitations, namely that

- although reduced, hypoglycemic episodes still occur; and

- the duration of action remains too short to provide sufficient 24-hour insulin supply in many patients. ${ }^{4}$

These limitations explain the need for a further search for new long-acting insulin analogues with a near-flat time-action profile providing adequate insulin supplementation during 24 hours. In this way, fewer injections are needed, undoubtedly leading to improvement of both quality of life and patient compliance. Finally, the new insulin analogue should be affordable and free from other complications, including weight gain.

\section{The Concept}

Insulin degludec (IDeg) is a new ultra-long-acting basal insulin analogue manufactured by Novo Nordisk currently in Phase III of clinical development. When compared to the human insulin amino acid sequence, IDeg is characterised by the deletion of ThrB30 and the addition of a 16-carbon fatty diacid attached to LysB29 via a glutamic acid spacer, explaining the official name LysB29 (Ne-hexadecandiyl-y-Glu)des(B30) human insulin (see Figure 1). This unique structure promotes the formation of multihexamers in the subcutaneous tissue. This feature is key to the long duration of action of the compound as resorption of insulin from the subcutaneous tissue at the injection site is dependent on molecular weight. This principle has been exploited when the short-acting insulin analogues were created, that are present in vial and at the injection site as monomeric insulines that move into the bloodstream almost instantaneously. The long acting insulin analogues also already exploited this principle, with detemir forming dihexamers and binding to albumin due to the presence of a free fatty acid side chain and glargine having a lower isoelectric point leading to precipitation at $\mathrm{pH} 7$ under the skin. The major difference between glargine and detemir and the new insulin, IDeg, lies in the stability of protraction. Indeed, protraction on the basis of precipitation is still very variable in release, whereas dihexamer and in particular multihexamer formation added to albumin binding gives extremely stable and reliable release patterns.

In the case of IDeg, extremely long multihexamers will be formed in the subcutaneous tissue, providing a buffering effect against changes in absorption rate. From these depots, IDeg monomers are subsequently released very slowly but continuously. This release and the subsequent binding of the monomers to albumin, in subcutaneous tissue as well as in the blood stream, both contribute to the ultra-long stable and peak-less pharmacokinetic (PK) profile of the molecule. Jonassen et al. showed that the steady-state PK profile, determined after six consecutive days of once-daily dosing with IDeg ( $5 \mathrm{nmol} / \mathrm{kg}$ ) in type 1 diabetes patients, demonstrated a stable exposure over 24 hours with a half-life ( $\left.t \frac{1}{2}\right)$ longer than 24 hours (see Figure 2). IDeg was detectable in the circulation for at least 96 hours after the final injection, although this finding not necessarily implicates a continuing biological activity at that time. 4.5 This prolonged profile opens new perspectives for insulin therapy. In contrast to our present way of using the basal analogues, where windows of timing when the insulin can be injected are quite narrow,
Figure 1: Molecular Structure of Long-acting Insulin Analogue - Insulin Degludec

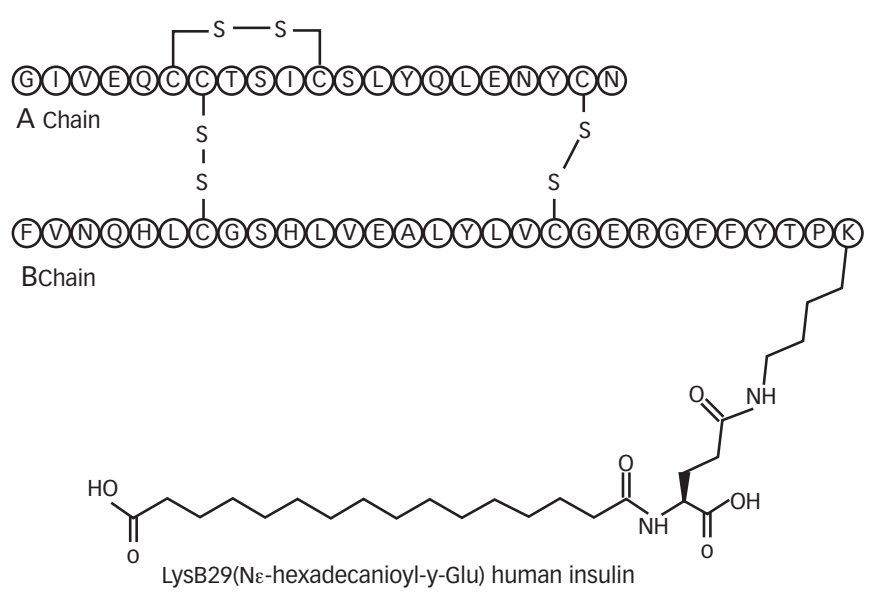

Figure 2: Mean Serum Insulin Degludec Concentration-time Profile in Steady State After Six Days ${ }^{5}$

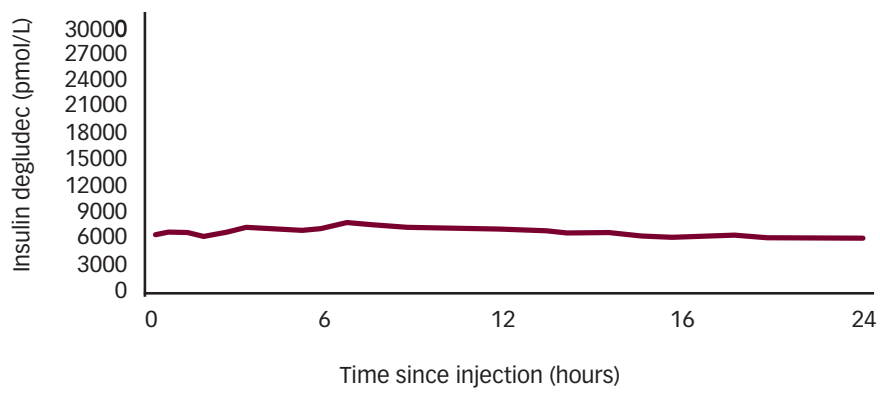

Mean serum insulin degludec concentraion-time profile in steady state following 6 days $(5.0 \mathrm{nmol} / \mathrm{kg}$, once daily).

\section{Figure 3: Ultra-Iong Acting Insulin - Very Flat Profile}

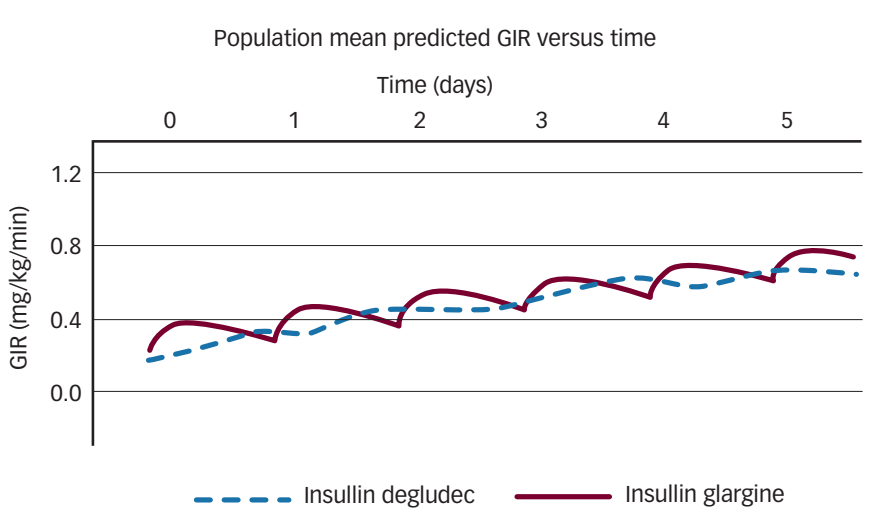

GIR $=$ glucose infection rate.

allowing day-to-day variation of injection timing only to last a few hours, IDeg might allow for more flexibility. Indeed, the ultralong profile will lead to the build-up of a plateau of circulating basal insulin, with, when injected every 24 hours, a topping-up of the plateau, whereas in the case of the other basal insulins, the levels of basal insulin will have dropped to very low or absent by the time the next injection is given (Figure 3).

Another advantage of the mechanism of protraction is as alluded to before, an enhanced stability in the release pattern, reflecting in less variability, a feature of crucial importance for type 1 diabetic patients, 
but also type 2 patients will benefit as variability contributes to hypoglycaemia risk. Heise et al. compared the pharmacodynamic (PD) properties of IDeg and insulin glargine in a randomised double-blind study in type 1 diabetes patients. They clearly showed significantly less within-subject variability with an evenly distribution over a 24-hour period (no differences between first and second 12 hours) in the IDeg group. ${ }^{6}$

These PK and PD studies provided an excellent and promising platform for further clinical trials, aiming to improve glycaemic control and lower hypoglycaemia risk compared to currently available insulin analogues. Another major benefit comprises the timing of injection. Based on the above-mentioned data, a wide interval of 24 to 48 hours between two consecutive injections is allowed, which will certainly increase patient compliance.

Another important asset of the new IDeg molecule is the fact that it can be combined with a monomeric short-acting analogue, with both insulins in the combination retaining their own profile of action. Thus, Novo Nordisk has created degludecplus, a combination of IDeg and aspart insulin (IASP). This is again a first of a new generation of 'combination insulins' rather than 'premixes', as in the premixes a clear interference between the different components of the mix can be observed, altering the action profiles of the individual components leading, for example, to the 'shoulder' effect, prolonging the action of the short-acting component, a feature not happening with degludecplus. ${ }^{5}$ This coformulation combined the simplicity of one insulin injection with the advantages of the more physiological profiles of both prandial and basal insulin analogue. This might be convenient not only for insulin initiation but also for intensification of insulin therapy because many patients will require prandial insulin on top of basal insulin therapy.

Finally, it is important to note that IDeg is a full agonist of the insulin receptor and maintains the metabolic responses of human insulin. IDeg has a low affinity for the human insulin-like growth factor-1 receptor (IGF-1 receptor), comparable with that of human insulin. ${ }^{4}$ In order to investigate metabolic responses and molecular safety Nishimura et al. performed binding studies using recombinant human insulin receptors and human IGF-1 receptors. The affinity of IDeg for human insulin receptor isoforms was found to be similar while the affinity for the human IGF-1 receptor was lower. In particular, IGF-1 receptor binding relative to insulin receptor binding was low $(<1 \%$ relative to human insulin) as well as was the mitogenic/metabolic potency ratio ( $<1 \%$ relative to human insulin). ${ }^{?}$

In parallel to the introduction of IDeg, several companies are also developing new longacting basal analogues (reviewed in simon et al. ${ }^{4}$ ). Several mechanisms of protraction are used, like PEGylation or modification of the formulation of existing long-acting analogues, like glargine. Clinical studies are still ongoing, preventing solid conclusions. Results are expected within the next two years. ${ }^{4}$

\section{The Promise}

The safety and efficacy of IDeg and degludecplus in patients with type 1 and 2 diabetes mellitus has been investigated in three published Phase II trials.

Birkeland et al. conducted a 16-week, randomised, open-label trial in type 1 diabetes patients who were treated with various insulin regimens during at least 12 months before inclusion and having a glycated haemoglobin $\left(\mathrm{HbA}_{1 \mathrm{c}}\right)$ of $7-10 \%$. Exclusion criteria included impaired renal and hepatic function, history of recurrent major hypoglycaemia, history of hypoglycaemia unawareness and pregnancy. Sixty participants were assigned according to a 1:1:1 randomisation to receive subcutaneous injections of IDeg $600 \mu \mathrm{mol} / \mathrm{L}$ (Group A; 1 Unit = $6 \mathrm{nmol}$ ), IDeg $900 \mu \mathrm{mol} / \mathrm{L}$ (Group B, 1 Unit $=9 \mathrm{nmol}$ ) or Insulin glargine (IGlar) $600 \mu \mathrm{mol} / \mathrm{L}$ (Group C, 1 Unit $=6 \mathrm{nmol})$. The injections were all administered once daily, approximately at the same time, preferably in the thigh and combined with Insulin aspart (IASP) at mealtimes, which was given preferably in the abdominal wall. At 16 weeks, no differences were observed in decrease of $\mathrm{HbA}_{1 \mathrm{c}}$ levels between the different groups with comparable mean total daily doses of insulin. The rates of confirmed hypoglycemia (defined as plasma glucose $<3.1 \mathrm{mmol} / \mathrm{L}$ ) and of nocturnal hypoglycaemia (between 23.00 and 05.59 hour) were significantly lower in the IDeg group A compared to IGlar. This trend was also seen in the IDeg group B versus IGlar, although here the difference was not statistical significant. Other adverse events including injection site reactions, body weight, and the occurrence of serum insulin-specific antibodies were not different in the three treatment groups. Taken together this trial demonstrates a better tolerability profile for IDeg group A. IDeg in combination with mealtime IAsp is a well-tolerated and efficacious treatment option in type 1 diabetes patients, with comparable glycaemic control to IGlar at comparable doses, but with lower rates of hypoglycaemia. Based on the results of this trial the company decided to discontinue the development of IDeg Group B formulation ( $9 \mathrm{nmol}$ ) and to proceed with IDeg Group A formulation (6 nmol) for subsequent Phase III trials. ${ }^{8}$

In a second Phase II trial Zinman et al. investigated the clinical efficacy and safety of IDeg in patients with insulin-naïve type 2 diabetes experiencing inadequate control with oral antidiabetic drugs. In this 16-week, randomised, open-label trial, 245 patients with $\mathrm{HbA}_{1 \mathrm{c}}$ levels between 7 and $11 \%$ were randomly allocated in a 1:1 ratio to receive IDeg once daily (Group A) or IGlar (Group C). On the basis of the PK and PD data, demonstrating the ultra-long duration of action, a third 'proof-of-concept' arm was added, where IDeg was injected three times a week (Group B), being Monday, Wednesday and Friday, with the weekends 'insulin-free'. All arms received insulin in combination with metformin. Similar to the results of the trial in diabetes type 1 patients no differences were seen at 16 weeks in decrease of $\mathrm{HbA}_{1 \mathrm{c}}$ between the three treatment groups. The number of hypoglycaemias was very low, but again the rate was lower - though no statistical significance was shown - in the IDeg once-daily group ( $8 \%$ formulate on A, $15 \%$ formulation B) compared to the two other groups, in which the hypoglycaemic rate was identical (23\%). The higher hypoglycaemic rate in the three-weekly regimen compared to the once-daily regimen of IDeg may be explained by an enhanced peak effect if a higher dose of IDeg is administered, as the starting dose in the former group was double that of the latter group. No trial product related serious adverse events were reported, whereas body weight was stable throughout the trial in each treatment group. ${ }^{9}$

In addition to IDeg, Novo Nordisk also produced a combination formulation combining IDeg and IAsp called degludecplus. In a recent 16-week, open-label Phase II trial Heise et al. investigated efficacy and safety of degludecplus, comprising $70 \%$ IDeg and $30 \%$ IAsp (Group A) or an alternative co-formulation (AF) comprising $55 \%$ IDeg and $45 \%$ 
IAsp (Group B) compared to IGlar (Group C) in insulin-naive type 2 diabetes inadequately controlled with oral antidiabetic drugs $\left(\mathrm{HbA}_{1 \mathrm{C}}\right.$ 7-11\%). Insulin was given before the evening meal and all participants also received metformin. Dose of the injection was titrated according to the fasting plasma glucose level. 178 patients were assigned according to a 1:1:1 randomisation. Once again there were no significant differences in reduction in $\mathrm{HbA}_{1 \mathrm{c}}$ between the three groups. Rates of hypoglycaemia were low with no differences between the treatment arms, although nocturnal hypoglycaemia occurred more in Group B. No treatment related serious adverse events and differences in body weight were reported. The authors concluded that once-daily degludecplus was safe, well tolerated with comparable overall glycaemic control and low rates of hypoglycaemia to IGlar, but with the additional benefit of better post-dinner plasma glucose control. In view of the finding of increased nocturnal hypoglycaemia, the clinical development of the alternative formulation has been discontinued. ${ }^{10}$

In conclusion, Phase II trials have shown that IDeg provides comparable glycaemic control compared to IGlar at similar unit doses in subjects with type 1 and type 2 diabetes. There were no differences in hypoglycaemic events between the two treatment groups in type 2 diabetes, whereas in type 1 diabetes patients the rate of confirmed and nocturnal hypoglycaemia was statistically significantly lower in the IDeg once-daily treatment group compared with IGlar once daily. The longer duration of action of IDeg may provide real 24-hour action with flexibility in dosing at any time of the day."

Currently, several Phase III trials, both in type 1 and type 2 diabetes patients, are registered at www.ClinicalTrials.gov (including approximately 7,000 participants), aiming to assess the efficacy and safety of IDeg. No full reports are available at present and only abstracts, presentations at conferences and press reports are available.

These preliminary results show overall that IDeg reached in all trials the non-inferiority criterion versus comparator (IGlar), with in many trials showing a clear advantage in hypoglycaemia risk, in particular in nocturnal hypoglycaemia risk. In the Phase III trials, stretching the principle of long duration of action as far as three-times weekly (which was demonstrated as proof of principle in Phase II) demonstrated not feasible in larger patient groups, with non-inferiority versus once daily IGlar not reached. However, several studies investigating 'flexibility' of dosing were included in the Phase II programme and did confirm the ultralong duration of action, allowing postponing of dosing without compromising glycaemic control (measured as $\mathrm{HbA}_{1 \mathrm{c}}$ and hypoglycaemia risk). The first full reports of these trials are awaited.

\section{Conclusion}

The development of insulin degludec, a new generation soluble basal insulin with an ultra-long peakless pharmacokinetic profile, and of degludecplus, a coformulation of insulin degludec and insulin aspart, has renewed the interest in insulin therapy for type 1 and 2 diabetes patients. Based on promising Phase II and preliminary Phase III data, insulin degludec has the potential to optimise glycaemic control, reduce hypoglycaemia and improve compliance by providing more flexible dosing schedules. The reduction in nocturnal hypoglycaemias really reflects the more stabile profile of this basal insulin, allowing tight titration of the glycaemia without increasing the risk of hypoglycaemia. In combination with a healthy lifestyle this new generation of insulin might become the new standard of care in diabetes mellitus reducing both fatal and non-fatal complications and improving quality of life. ${ }^{12}$
1. Action to Control Cardiovascular Risk in Diabetes Study Group, Effects of intensive glucose lowering in type 2 diabetes, New Engl I Med, 2008;358:2545-59.

2. Duckworth W, Abraira C, Moritz T, et al., Glucose control and vascular complications in veterans with type 2 diabetes, New Eng/ J Med, 2009;360:129-39.

3. ADVANCE Collaborative Group, Intensive blood glucose controle and vascular outcomes in patients with type 2 diabetes, New Eng/ I Med, 2008;358(24):2560-72.

4. Simon AC, Devries $\mathrm{JH}$, The future of basal insulin supplementation, Diabetes Technol Ther, 2011:13(Suppl. 1):S103-8.

5. Jonassen I, Havelund S, Ribel U, et al., Insulin degludec: multi-hexamer formation is the underlying basis for this new generation ultra-long acting basal insulin, Diabetologia 2010;53(Suppl. 1):S388[abstract 972].

6. Heise T, Hermanski L, Nosek L, et al., Insulin degludec: less pharmacodynamic variability than insulin glargine under steady state conditions, Diabetologia, 2010;53(Suppl. 1):S387[abstract 971].

7. Nishimura E, Sorensen AR, Hansen BF, et al., Insulin degludec: a new ultra-long, basal insulin designed to maintain full metabolic effect while minimizing mitogenic potential, Diabetologia, 2010;53(Suppl. 1):S388[abstract 974].

8. Birkeland $\mathrm{KI}$, Home PD, Wendisch U, et al., Insulin Degludec in Type 1 Diabetes: A randomized controlled trial of a newin Type 1 Diabetes: A randomized controlled trial of a new-
generation ultra-long-acting insulin compared with insulin glargine, Diabetes Care, 2011;34(3):661-5.
9. Zinman B, Fulcher G, Rao PV, et al., Insulin Degludec, an ultra-long-acting basal insulin, once a day or three times a week versus insulin glargine once a day in patients with type 2 diabetes: a 16-week, randomised, open-label, phase 2 trial, Lancet, 2011;377:924-31

10. Heise T, Tack CJ, Cuddihy R, et al., A new generation ultralong- acting basal insulin with a bolus boost compared with insulin glargine in insulin-naïve people with type 2 diabetes: a randomized, controlled trial, Diabetes Care, 2011:34(3):669-74.

11. Little $S$, Shaw J, Home P, et al., Hypoglycemia rates with basal insulin analogs, Diabetes Technol Ther, 2011:13(Suppl. 1):S53-64.

12. Kudva YC, Basu A, Ultra-long-acting insulins for a lifestylerelated pandemic, Lancet, 2011;377:924-31. 